Research Article

\title{
Cross-Cultural Communication of Regional Images Based on Multimodal Discourse Analysis and Network Security
}

\author{
Qing Xu $\mathbb{D}^{1}$ and Guangming Wang $\mathbb{D}^{2}$ \\ ${ }^{1}$ Wenzhou University College of Foreign Studies, Wenzhou University, Wenzhou, Zhejiang Province 325035, China \\ ${ }^{2}$ Zhengzhou University, School of Politics and Public Administration, Zhengzhou, Henan Province 450001, China \\ Correspondence should be addressed to Qing Xu; xuqing@wzu.edu.cn
}

Received 23 March 2021; Revised 15 April 2021; Accepted 25 April 2021; Published 7 May 2021

Academic Editor: Mian Ahmad Jan

Copyright (C 2021 Qing Xu and Guangming Wang. This is an open access article distributed under the Creative Commons Attribution License, which permits unrestricted use, distribution, and reproduction in any medium, provided the original work is properly cited.

\begin{abstract}
Numerous evaluation methods are used as effective indicators for the cross-cultural communication of a regional image. However, because these indicators are primarily in a foreign cultural situation, it is challenging to measure their attitudes and cognitions across geographic distances and cultural differences. This challenge motivated us to combine cultural communication methods and modern location service technology to carry out regional image communication research. First, this article examines the basic process of cross-cultural communication and the generation of regional images from an information transmission and network security perspective. It combines multimodal discourse to analyze influencing factors and establishes a hierarchical model of the cross-cultural communication indicator system. Secondly, based on the location-based service (LBS), this article adds scene information to develop a user tendency model. This model effectively handles the conversion between two-dimensional scoring space and three-dimensional tendency space and can adapt to the short-term changes of users' tendency. The experimental results verify that the location-based e-commerce recommendation algorithm proposed in this paper can better solve information overload and obtain a significant recommendation effect.
\end{abstract}

\section{Introduction}

The division of physical space makes it possible to produce a regional image, and it also creates a space of different ideas for the researchers $[1,2]$. The cross-cultural communication path aims to explore how to bridge the division of physical space through the construction of information space and adjust the conceptual space solidified by different communication subjects with the provisioning of network security [2]. After the spatialization of philosophical and sociological research, the academic circles have been lacking in studying foreign land's image from the perspective of space, which is precisely a critical issue that is indispensable in the study of foreign images $[3,4]$. The problem of the foreign image was initially the problem of space and then the image [4].

In recent years, relevant scholars have carried out research around the aforementioned issues [4]. Wan-Shilu
Yang and Hung-Chi Chen [5] compared the information keywords by initially calculating the similarity between the content of each web page and the user's interest, then introduced the location information, and constructed the utility function. Finally, they calculated the result and sort the web recommendation list. In [6], the authors have carried out research on the application of dynamic user analysis in mobile services by providing relevant services and information based on a new user's preferences. In [7], the authors incorporated location and other contextual attributes into the common user interest model, further enriching the user interest model. For each scenario, an associated user interest needs to be generated $[8,9]$. Therefore, the provision of services is suitable for the user's interest and can be better applied to the user's current scenario [10]. Reasonable evaluation indicators and methods are an effective indicator of the cross-cultural communication elements of a regional image. However, because the 
survey subjects are in a foreign cultural situation, it is extremely difficult to measure their attitudes and cognitions across geographic distances and cultural differences. Hence, this article combines cultural communication methods and modern location service technology to carry out regional image communication [11-13].

The main contributions of this work are as follows:

(1) First, this article introduces the research overview of location services and cross-cultural communication of geographical image

(2) Next, we discuss the evaluation index analysis methods based on location services and analytic hierarchy from network security viewpoint

(3) Finally, we conduct a simulation test on the locationbased and service-based cross-cultural communication model, which will be established in this paper

The rest of this paper is organized as follows. In Section 2 , a detailed literature review of related studies is provided. In Section 3, image evaluation based on location service and level analysis is discussed. In Section 4, the simulation test results on the proposed approach are discussed. Finally, we conclude the paper and provide future research directions in Section 5 .

\section{Related Work}

In this section, first, we discuss the existing literature pertaining to location-based service (LBS) followed by crosscultural communication of geographical images. This will lay a solid foundation for our proposed work and objectives.

2.1. Location-Based Service. Location-based service (LBS) technology integrates Internet, wireless communication, mobile positioning, and GIS [14-16]. As mobile phones become an indispensable part of our lives, the mobile service market has an increasingly urgent need for using mobile phones to implement location information. An example of a recommendation algorithm combining location and network security is shown in Figure 1.

LBS can use the software and hardware resources of the telecommunication operator to obtain the geographic location information of the mobile terminal user. For example, LBS can be used for obtaining geographic location coordinates. Next, collaborative filtering is used at each layer (where the user is located) to obtain multiple recommendation lists. Finally, LBS weighs the recommendation lists of each layer to obtain the final recommendation list. With the support of GIS (geographic information system) platform, it can bring about economic growth of corresponding services.

\subsection{Cross-Cultural Communication of Geographical Image.}

The cognition of the external world is inseparable from the division of interior and exterior centered on the environment in which the cognition subject lives $[17,18]$. The problem of image actually involves the issue of the space between the cognition subject and the image itself. One of the sources of people's cognition of space problems is caves. One of the most primitive forms of human settlements is caves. In Chinese myths and legends, primitive humans before the Chao family lived in naturally formed caves $[19,20]$. The cave separates two completely heterogeneous spaces inside and outside with solid objects and possesses the most primitive functions of all spaces: belonging and separation. Physical separation also means separation in culture, identity, and cognitive norms. This separation treats the subject's culture and norms as authentic, conceals a certain truth, and establishes the subject's cognitive hegemony through the establishment of rules for the inhabitants of the space to understand the outside world [21-23]. The existence of this hegemony strengthens the spiritual space of the subject and makes it possible to separate all the spiritual activities of the subject (including the construction of the images) from the original face of the world. In other words, after geographical space divides the "old and dead" foreign country into two isolated and closed entities, imagine it as the only way to fill the "picture in the mind." The information spaces interact with physical space to continuously adjust the cognitive space of human beings [24]. Therefore, the route of cross-cultural dissemination of regional images is to study how the communication media, which is the connection link, create an information space and use their respective conceptual spaces as the scale of communication to build a cognitive relationship between the interacting parties.

\section{Image Evaluation Based on Location Service and Cross-Cultural Communication}

In this section, first, we discuss the location service model of our proposed approach followed by the cross-cultural communication of regional images based on multimodal discourse analysis and network security.

3.1. Location Service Model. In order to satisfy the different preferences of users in different regions, various researchers have made attempts, and the most famous one is the LARS model, which uses traditional collaborative filtering at different spatial levels to recommend users with spatial attributes $[25,26]$. Projects without spatial attributes have the characteristics of high recommendation accuracy and high system stability. However, the user's spatial location distribution affects the recommendation system. Moreover, from the perspective of a larger spatial scale, the interests of users are different in different countries and regions. For each scenario, an associated user interest will be generated $[8,9]$. Therefore, the provision of services is suitable for the user's interest and can be better applied to the user's current scenario [10]. Reasonable evaluation indicators and methods are an effective indicator of the cross-cultural communication elements of a regional image. It is subdivided into spatial scale of the city level; users in different regions also have different interests and tastes, and they will also show different preferences for different regional images [27, 28]. 


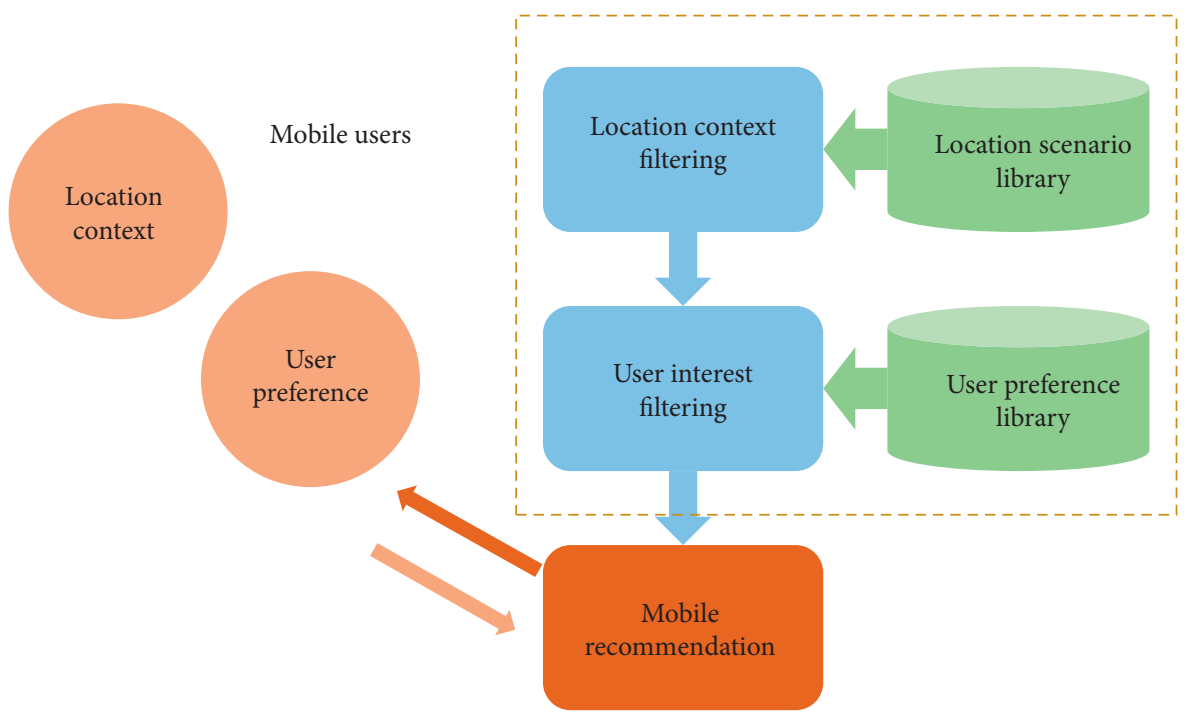

FIGURE 1: An example of a recommendation algorithm combining location and network security.

In this paper, we consider the similarity of the user distribution of the project based on the idea that if two projects are similar, then their user distributions are similar to each other. We propose an indicator called the distance distribution coefficient to measure the user distribution of a given project. This article selects the best degree of competition for tasks in different locations. The minimum positioning objective function corresponding to the optimal radius is

$$
\min _{w, b, e} \varphi(a, b, e)=\frac{1}{2} \omega^{T} \omega+\frac{1}{2} \gamma \sum_{k=1}^{N} e_{k}^{2} .
$$

The constraints are

$$
y_{k}\left[\omega^{T} \phi\left(x_{k}\right)+b\right]=1-e_{k}, \quad k=1,2, \ldots, N .
$$

The LaGrange function is defined as

$$
L(w, b, e, \alpha)=\phi(w, b, e)-\alpha_{k} \sum_{k=1}\left\{\alpha_{k} y_{k}\left[\omega^{T} \phi\left(x_{k}\right)+b\right]-1-e_{k}\right\}
$$

The above equation is optimized, and the matrix equation can be finally obtained as follows:

$$
\left[\begin{array}{cc}
0 & -Y^{T} \\
Y & Z Z^{T}+\gamma^{-1} I
\end{array}\right]\left[\begin{array}{l}
b \\
\alpha
\end{array}\right]=\left[\begin{array}{l}
0 \\
I
\end{array}\right] .
$$

Bringing in the Mercer condition, the final decision function is obtained as

$$
y(x)=\operatorname{sgn}\left[\sum_{k=1}^{N} \alpha_{k} y_{k} \psi\left(x_{k}, x_{l}\right)\right] .
$$

After the user distance distribution coefficient is obtained, the similarity calculation between items can be similar based on the network structure:

$$
\max _{\left\{p_{i}, y_{i}\right\}} \pi(p, y, x)=\sum_{j=1}^{M} \sum_{i=1}^{K_{B(I)}}\left(p_{i}-c_{i}\right) x_{i j} y_{i} .
$$

In this paper, the similarity using the distance distribution coefficient is called geographic similarity, and the cosine-like similarity calculation method of geographic similarity is as follows:

$$
\begin{aligned}
\text { s.t. } p_{i} & >c_{i}, \\
y_{i} & >x_{i j}, \\
y_{i} & =0 \text { or } 1 \quad\left(i=1,2, \ldots, K_{B(I)}, j=1,2, \ldots, M\right) .
\end{aligned}
$$

After obtaining the mixed similarity of the fusion location factors, the users are divided into levels of different heights by using a hierarchical structure according to the user's location coordinate information, as shown in equations (8) and (9):

$$
\begin{aligned}
& \max _{\left\{x_{i j}\right\}} \mu_{j}(x)=\sum_{i=1}^{K_{B(I)}} r_{i j} x_{i j}, \\
& \text { s.t. }\left(r_{i j}-p_{i}\right) x_{i j} \geq 0, \\
& x_{i j} \leq y_{i}, \\
& x_{i j}=0 \text { or } 1 \quad\left(i=1,2, \ldots, K_{B(I)} ; j\right. \\
& =1,2, \ldots, M) .
\end{aligned}
$$

Next, we perform collaborative filtering at each layer (where the user is located) to obtain multiple recommendation lists; finally, weigh the recommendation lists of each layer to obtain the final recommendation list [29]. After the spatialization of philosophical and sociological research, the academic circles have been lacking in the study of the image of foreign land from the perspective of space, and this is precisely an important issue that is indispensable in the 
study of foreign image. However, because the survey subjects are in a foreign cultural situation, it is very difficult to measure their attitudes and cognitions across geographic distances and cultural differences. Based on this, this article combines cultural communication methods and modern location service technology to carry out regional image communication research. The problem of the foreign image is firstly the problem of space and then the problem of the image. The specific process of the recommendation list will be shown in Figure 2.

In order to improve the accuracy of the recommendation system, this paper adds the user distribution of the item as a feature parameter to the calculation of the recommendation algorithm on the basis of the traditional method that only relies on the score data to calculate the similarity. After the hybrid similarity is obtained through the improved similarity calculation, the clustering idea is used to divide and generate the geographical user clusters, and then, the collaborative filtering recommendation is performed. The main contributions are as follows. After the first division is completed, traverse the user data in the area to obtain the user item score matrix of each layer of the area, and calculate the mixed similarity of the fusion geographic factors according to the similarity calculation method introduced above degree. Finally, a global recommendation list and several local recommendation lists are obtained.

3.2. Cross-Cultural Communication Evaluation Based on Analytic Hierarchy Process. After the hybrid similarity is obtained through the improved similarity calculation, the clustering idea is used to divide and generate the geographical user clusters, and then, the collaborative filtering recommendation is performed. Therefore, the provision of services is suitable for the user's interest and can be better applied to the user's current scenario. Reasonable evaluation indicators and methods are an effective indicator of the cross-cultural communication elements of a regional image. Subdivided into the spatial scale of the city level, users in different regions also have different interests and tastes, and they will also show different preferences for different regional images.

3.2.1. The Establishment of Hierarchical Analysis Structure. First, perform collaborative filtering in each layer where the user is located to obtain multiple recommendation lists; finally, weigh the recommendation lists of each layer to obtain the final recommendation list. By exploring the potential impact of cross-cultural communication assessment, this article has identified eight influencing factors, and the established hierarchical analysis structure is shown in Figure 3.

3.2.2. The Construction of the Judgment Matrix. After the first division is completed, the user data of the area is traversed to obtain the user item score matrix of each layer in the area, and the similarity calculation method is introduced based on the above degree. We can find that the geographical similarity value in the average ranking score is smaller than the cosine similarity, and the accuracy and recall rate are higher than the cosine similarity. This shows that the recommendation effect of the geographical similarity is better than the traditional cosine similarity, which shows that the user location distribution can play an effective role in project-based collaborative filtering. Table 1 shows the matrix results based on the data items.

3.2.3. Matrix Consistency Check.

$$
M_{i}=\prod_{j=1}^{n} a_{i j}, \quad i=1,2, \ldots, n .
$$

(1) Calculate the $n^{\text {th }}$ root:

$$
\begin{aligned}
\bar{W}_{i} & =\sqrt[n]{M_{i}}, \\
W_{i} & =\frac{\bar{W}_{i}}{\sum_{j=1}^{n} \bar{W}_{j}} .
\end{aligned}
$$

(2) Calculate the largest characteristic root of the judgment matrix:

$$
\lambda_{\max }=\sum_{i=1}^{n} \frac{(A W)_{i}}{n W_{i}}
$$

Through the calculation of each level of the judgment matrix, Table 2 shows the judgment matrix results based on the data items.

\section{Model Effect and Simulation Test Results}

In this section, first, we provide the evaluation index of our model followed by model test data and the ultimate experimental results.

4.1. Model Evaluation Index. Earlier, we discussed the evaluation index analysis methods based on location services and analytic hierarchy. This study uses precision to measure the prediction accuracy of the generated recommendation list [30]. The accuracy is defined as follows:

$$
\text { Precision }=\frac{\sum_{u \varepsilon U}|R(u) \cap T(u)|}{\sum_{u \varepsilon U}|T(u)|} .
$$

At the same time, we have also carried out the calculation and comparison of the recall rate (recall), which is defined as follows:

$$
\text { Recall }=\frac{\sum_{u \varepsilon U}|R(u) \cap T(u)|}{\sum_{u \varepsilon U}|R(u)|},
$$

where $T(u)$ is the list of items that the user actually likes in the test set. 


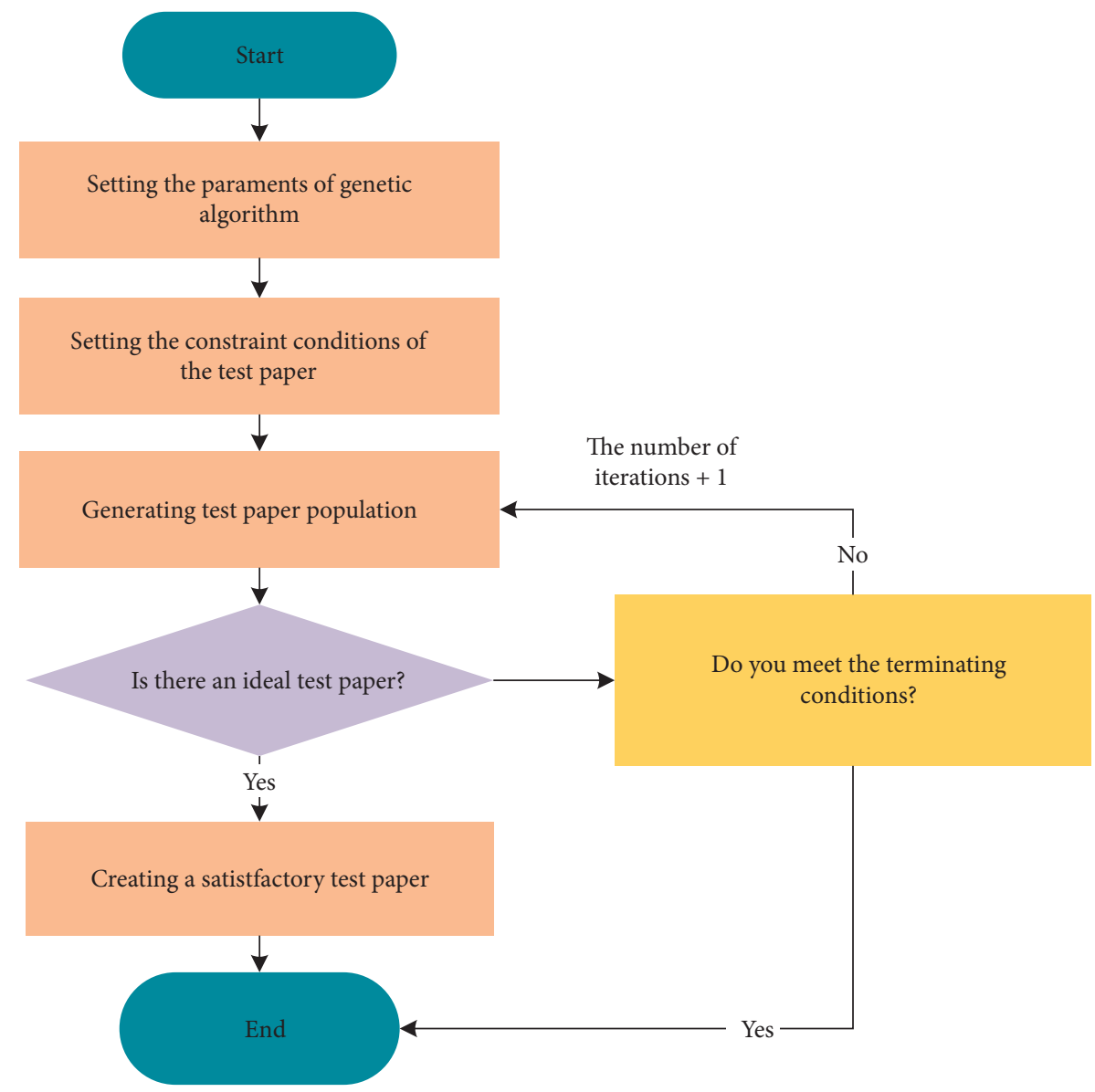

Figure 2: The specific process of the recommendation list.

$$
r_{i}=\frac{L_{i}}{N}
$$

In order to satisfy the broad interests of users, this study uses diversity indicators, namely, internal diversity and external diversity. Interuser diversity is measured by hamming distance, which is defined as follows:

$$
H_{u t}(L)=1-\frac{Q_{u t}(L)}{L} \text {. }
$$

Take the average of all users to get $H(L)$ of the system. As $H(L)$ increases, the diversity of the recommendation system increases.

And, the internal diversity of user $u$ (intrauser diversity) is defined as

$$
I_{u}(L)=\frac{1}{L(L-1)} \sum_{\alpha \neq \beta} \operatorname{sim}(\alpha, \beta) .
$$

4.2. Model Test Data. In the system testing phase, this article will conduct a simulation test on the location-based servicebased cross-cultural communication model established in this article. The dataset uses Gowalla data in the public database. Gowalla is a website that provides location checkin. This article uses the Gowalla dataset, which contains
30,367 geographic reference points in a certain area, as well as information about 357,753 visits by 19,183 users of Gowalla. We selected at least 20 users to visit each location, and each user visited at least 20 locations and finally obtained 85,862 times of 1962 users to 2063 locations [31]. Figure 4 shows a schematic diagram of partial location information of the database.

In the experiment designed in this article, each dataset is randomly divided, $80 \%$ of which is used for training. The training set is regarded as known information, and the test set does not allow any information to be used for prediction. According to the test set, the performance of this algorithm is evaluated from various aspects.

4.3. Model Test Results. This paper compares the three different similarity calculation forms, namely, cosine similarity, Jaccard similarity, and Sorensen similarity, under the combination of geographic similarity and traditional similarity under the change of the balance factor. The LARS introduced in paper is used for comparison. Although the LARS algorithm is not the best algorithm in the locationaware recommendation system, it takes into account the accuracy of the recommendation system and the stability in the actual system application. It is still a very practical algorithm. 


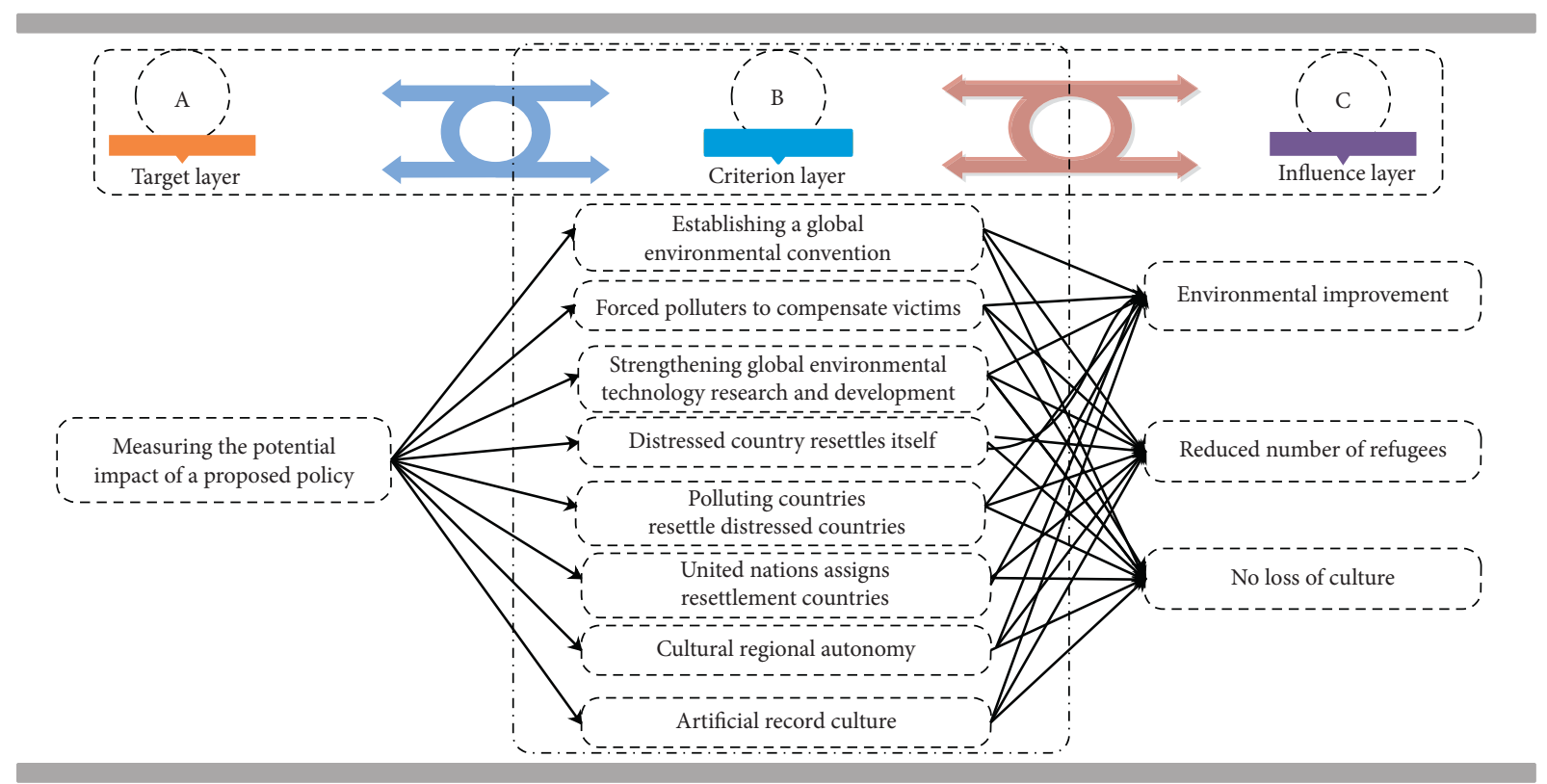

FIGURE 3: A hierarchical analysis structure for measuring the potential impact of cross-cultural communication assessment.

TABLE 1: The matrix results based on the data items.

\begin{tabular}{ccccccccc}
\hline$A$ & $B_{1}$ & $B_{2}$ & $B_{3}$ & $B_{4}$ & $B_{5}$ & $B_{6}$ & $B_{7}$ \\
\hline$B_{1}$ & 1 & $1 / 3$ & $1 / 3$ & $1 / 3$ & $1 / 4$ & $1 / 5$ & $1 / 3$ \\
$B_{2}$ & 3 & 1 & 2 & $1 / 3$ & $1 / 4$ & $1 / 5$ & $1 / 3$ \\
$B_{3}$ & 3 & $1 / 2$ & 1 & $1 / 3$ & $1 / 4$ & $1 / 5$ & $1 / 2$ \\
$B_{4}$ & 3 & 3 & 3 & 1 & $1 / 3$ & $1 / 4$ & 3 \\
$B_{5}$ & 4 & 4 & 4 & 3 & 1 & $1 / 3$ & $1 / 3$ \\
$B_{6}$ & 5 & 5 & 3 & 2 & $1 / 3$ & $1 / 3$ & $1 / 3$ & 3 \\
$B_{7}$ & 3 & 3 & 3 & $1 / 3$ & $1 / 3$ & $1 / 3$ & 1 \\
$B_{8}$ & 4 & 3 & & 3 & & 3 & $1 / 3$ \\
\hline
\end{tabular}

TABLE 2: The judgment matrix results based on the data items.

\begin{tabular}{|c|c|c|c|c|c|c|c|c|c|c|c|c|}
\hline \multicolumn{9}{|c|}{ Calculation result of judgment matrix } & $\lambda_{\max }$ & $\mathrm{CI}$ & RI & $\mathrm{CR}$ \\
\hline \multirow{10}{*}{$\mathrm{A} W=$} & \multirow{10}{*}[\begin{array}{l}{0.0328}\\
{0.0542}\\
{0.0478}\\
{0.1465}\\
{0.2142}\\
{0.3071}\\
{0.1083}\\
{0.0891}\end{array}]{} & \multirow{3}{*}{$B_{1}$} & \multirow{3}{*}{$W=$} & {$[0.6144]$} & \multirow{3}{*}{$B_{5}$} & \multirow{3}{*}{$W=$} & \multirow{3}{*}[\begin{array}{l}{0.1365}\\
{0.6250}\\
{0.2385}\end{array}]{} & \multirow{3}{*}{$\begin{array}{l}B_{1} \\
B_{2}\end{array}$} & \multirow{3}{*}{$\begin{array}{l}3.0735 \\
3.0536\end{array}$} & \multirow{3}{*}{$\begin{array}{l}0.0368 \\
0.0268\end{array}$} & \multirow{3}{*}{$\begin{array}{c}0.5200 \\
0.52\end{array}$} & \multirow{3}{*}{$\begin{array}{l}0.0707 \\
0.0515\end{array}$} \\
\hline & & & & 0.2684 & & & & & & & & \\
\hline & & & & {$[0.1172]$} & & & & & & & & \\
\hline & & \multirow{2}{*}{$B_{2}$} & \multirow{2}{*}{$W=$} & {$[0.5278]$} & \multirow{2}{*}{$B_{6}$} & \multirow[b]{2}{*}{$W=$} & {$[0.1168]$} & $B_{3}$ & 3.0618 & 0.0197 & 0.100 & 0.0478 \\
\hline & & & & $\begin{array}{l}0.3325 \\
01396\end{array} \mid$ & & & 0.6833 & $B$ & & & 05000 & 00036 \\
\hline & & \multirow{3}{*}{$B_{3}$} & \multirow{3}{*}{$W=$} & {$[0.5469]$} & \multirow{3}{*}{$B_{7}$} & \multirow{3}{*}{$W=$} & {$[0.1220]$} & $D_{4}$ & & & & \\
\hline & & & & 0.3445 & & & 0.3196 & $B_{5}$ & 3.0183 & 0.0091 & 0.5300 & 0.0176 \\
\hline & & & & {$[0.1085]$} & & & {$[0.5584]$} & $B_{6}$ & 3.0246 & 0.0123 & 0.5200 & 0.0236 \\
\hline & & \multirow{2}{*}{$B_{4}$} & \multirow{2}{*}{$W=$} & {$\left[\begin{array}{l}0.1095 \\
0.5816\end{array}\right]$} & \multirow{2}{*}{$B_{8}$} & \multirow{2}{*}{$W=$} & {$\left[\begin{array}{l}0.0936 \\
0.2797\end{array}\right]$} & $B_{7}$ & 3.0183 & 0.0091 & 0.4900 & 0.0178 \\
\hline & & & & {$[0.3090]$} & & & {$[0.6267]$} & $B_{8}$ & 3.0857 & 0.0428 & 0.4900 & 0.0825 \\
\hline
\end{tabular}

C-CF, J-CF, and S-CF appearing below are collaborative filtering based on user-item score data for cosine similarity, Jaccard similarity, and Sorensen similarity calculations. C-LARS, J-LARS, and S- LARS use three similarities for collaborative filtering calculations under the LARS algorithm. DC-CF, DJ-CF, and DS-CF are algorithms that calculate the hybrid similarity under three similarity forms and then perform collaborative filtering. DC-LARS,
DJ-LARS, and DS-LARS are algorithms based on user location distribution proposed in this paper. In the case of similarity calculation, NBI is a recommendation algorithm based on a bipartite graph network. Accuracy of cosine-like form in cosine similarity and geographic similarity are shown in Figures 5-7.

In Figure 5-7, Method $\mathrm{A}$ is the cosine-like method of geographic similarity, and Method B is the traditional cosine 


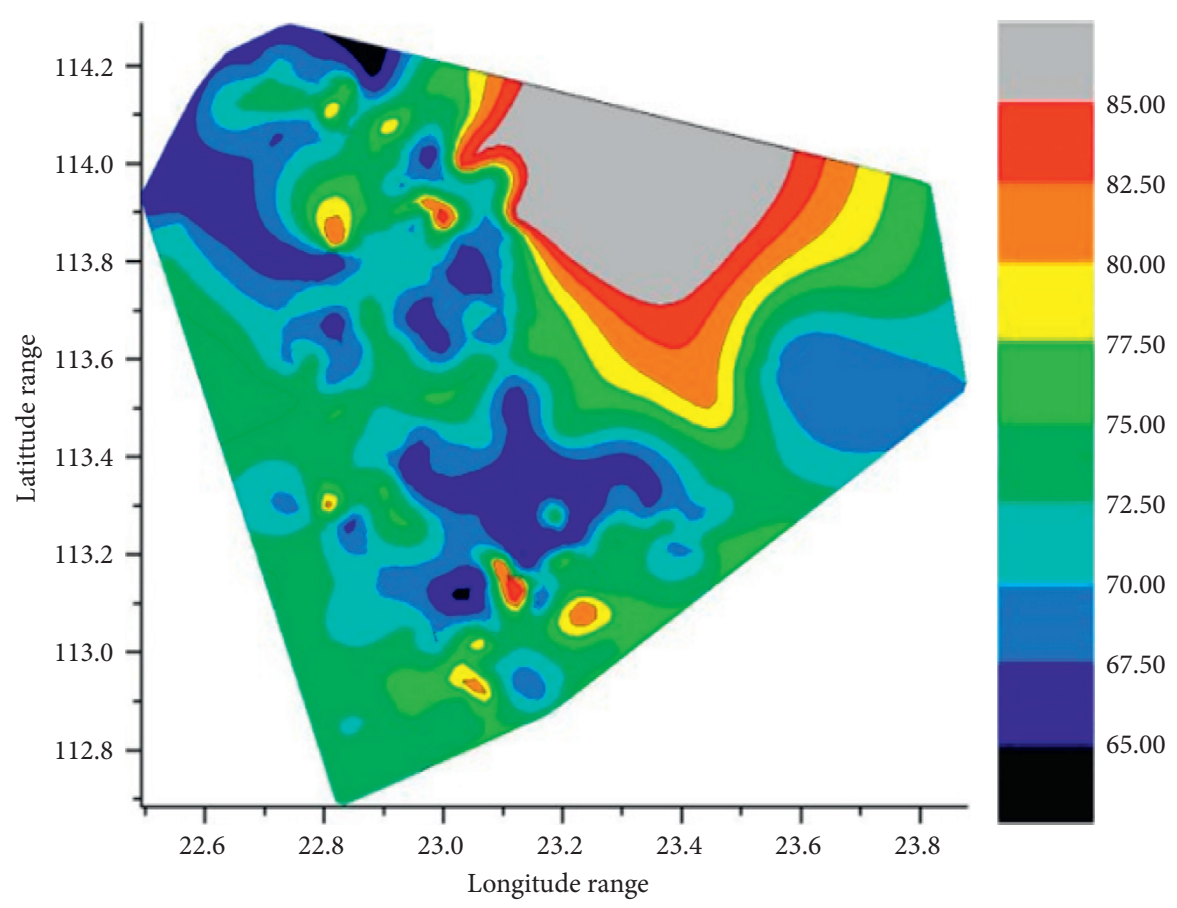

FIgURE 4: Schematic diagram of partial location information of the database.

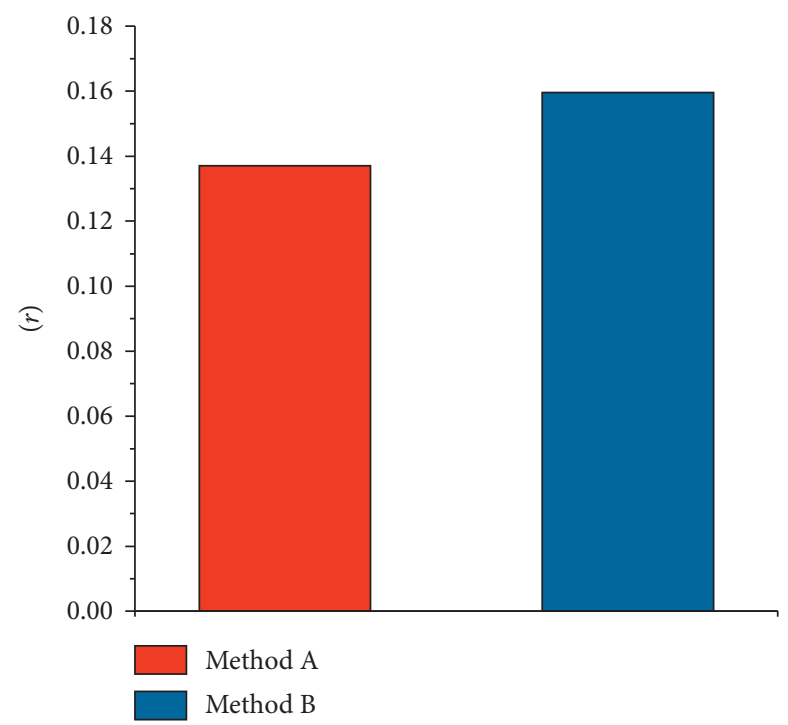

FIGURE 5: Accuracy of cosine-like form in cosine similarity and geographic similarity (r).

similarity method. The ordinate in the figure is the algorithm index; from left to right are the average ranking score, accuracy, and recall rate. As shown in Figure 5-7, we compared the performance of the cosine form of item-based collaborative filtering and the cosine-like form of geographic similarity in the dataset under the three indicators of average ranking score, accuracy, and recall rate. We can find that the geographical similarity value in the average ranking score is smaller than the cosine similarity, and the accuracy and recall rate are higher than the cosine similarity. This shows that the recommendation effect of the geographical similarity is better than the traditional cosine similarity, which reflects that the user location distribution can play an effective role in project-based collaborative filtering. 


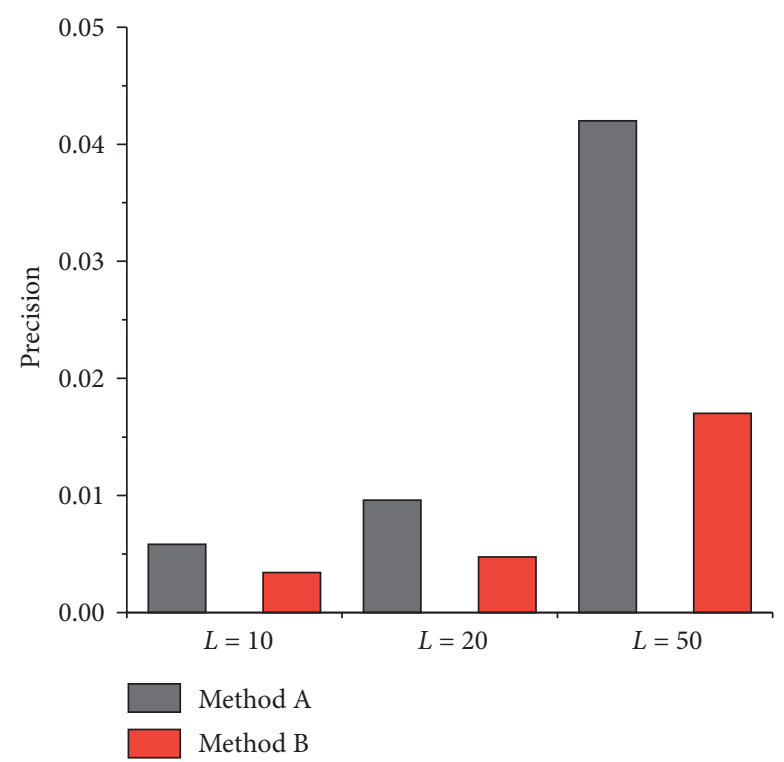

Figure 6: Accuracy of cosine-like form in cosine similarity and geographic similarity (Precision).

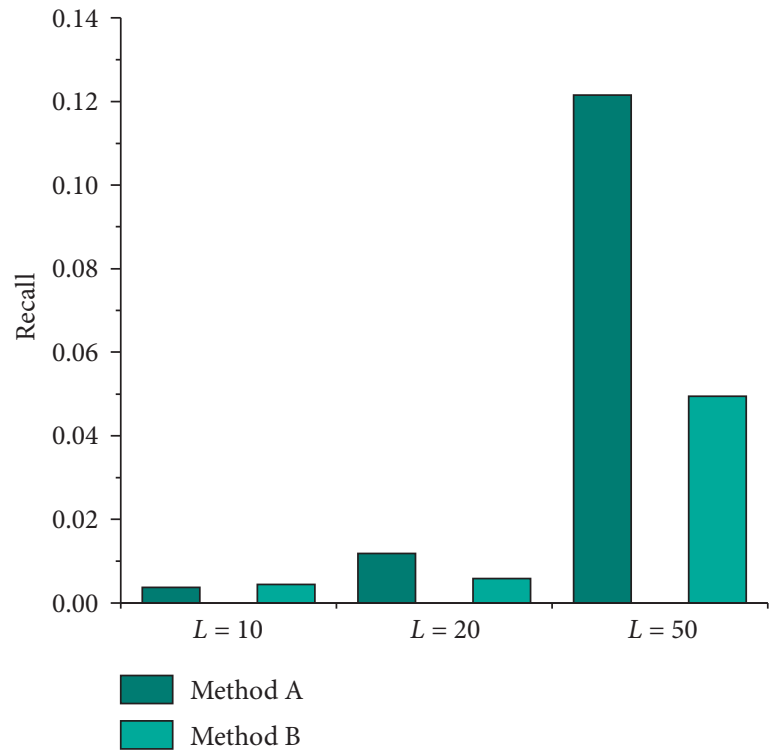

FIGURE 7: Accuracy of cosine-like form in cosine similarity and geographic similarity (Recall).

\section{Conclusion}

After the spatialization of philosophical and sociological research, the academic circles have been lacking in the study of the image of foreign land from the perspective of space, and this is precisely an important issue that is indispensable in the study of the foreign image. The problem of foreign image is firstly the problem of space and then the problem of the image. Reasonable evaluation indicators and methods are an effective indicator of the cross-cultural communication elements of a regional image. However, because the survey subjects are in a foreign cultural situation, it is very difficult to measure their attitudes and cognitions across geographic distances and cultural differences. This article combines cultural communication methods and modern location service technology to carry out regional image communication research. Due to the limited research conditions of this article, the research on the geographical image communication based on location services is not perfect. In the future, we will continue to devote ourselves to related research and to improve the progress of cross-cultural communication of regional image. 


\section{Data Availability}

The data used to support the findings of this study are available from the corresponding author upon request.

\section{Conflicts of Interest}

The authors declare that they have no conflicts of interest.

\section{Acknowledgments}

This study was supported by the project of Zhejiang Education Department: Regional Images in the MOOC and Multimodal Construction and Cross-cultural Communication (Y201941084).

\section{References}

[1] Z. Liu, "A study on English translation of tourism publicity in coastal cities from the perspective of cross-cultural communication," Journal of Coastal Research, vol. 115, no. sp1, p. 87, 2020.

[2] D. Warad, V. Rodriguez, and A. Nageswara Rao, "Crosscultural communication in pediatric oncology: catch them when they're young," Cancer, vol. 124, no. 7, pp. 1516-1517, 2018.

[3] M. F. Ilowite, T. I. Kang, and J. W. Mack, "Reply to Crosscultural communication in pediatric oncology: catch them when they're young," Cancer, vol. 124, no. 7, p. 1517, 2018.

[4] S. H. Kim, N. Petard and J. H. Hong, What is lost in translation: a cross-cultural study to compare the concept of nuttiness and its perception in soymilk among Korean, Chinese, and Western groups," Food Research International, vol. 105, pp. 970-981, 2018.

[5] K. Würth, W Langewitz, S Reiter-Theil, and S Schuster, “Their view: difficulties and challenges of patients and physicians in cross-cultural encounters and a medical ethics perspective," Bmc Medical Ethics, vol. 19, no. 1, p. 70, 2018.

[6] L. R. Koenig, M. Li, L. A. Zimmerman et al., "Associations between agency and sexual and reproductive health communication in early adolescence: a cross-cultural, cross-sectional study," Journal of Adolescent Health, vol. 67, no. 3, pp. 416-424, 2020.

[7] H. Haragus, R. Prejbeanu, J. Patrascu et al., "Cross-cultural adaptation and validation of the Romanian oxford shoulder score," Medicine, vol. 97, no. 23, p. e10926, 2018.

[8] Z. Wang, L. Gao, and J. Huang, "Location-flexible mobile data service in overseas market," IEEE Transactions on Mobile Computing, vol. 99, 2020.

[9] R. S. Thoma, A. Schwind, P. Wendland et al., "Cooperative passive coherent location: a promising $5 \mathrm{G}$ service to support road safety," IEEE Communications Magazine, vol. 57, no. 9, pp. 86-92, 2019.

[10] D. C. Turkoglu and M. E. Genevois, "A comparative survey of service facility location problems," Annals of Operations Research, vol. 292, 2020.

[11] I. Averbakh and W. Yu, "Multi-depot traveling salesmen location problems on networks with special structure," Annals of Operations Research, vol. 286, 2020.

[12] L. Navarro, "Injury frequency and characteristics (location, type, cause and severity) differed significantly among athletics ('track and field') disciplines during 14 international championships (2007-2018): implications for medical service planning," British Journal of Sports Medicine, vol. 54, no. 3, p. 159, 2020.

[13] J. H. Park, "Location of arrest and effect of prehospital advanced airway management after emergency medical servicewitnessed out-of-hospital cardiac arrest: nationwide observational study," Emergency Medicine Journal, vol. 36, no. 9, pp. 541-547, 2019.

[14] H. Andersson, "Using optimization to provide decision support for strategic emergency medical service planning three case studies," International Journal of Medical Informatics, vol. 133, pp. 103975.1-103975.10, 2020.

[15] C. Hansen, C. Mena, and E. Aktas, "The role of political risk in service offshoring entry mode decisions," International Journal of Production Research, vol. 57, 2019.

[16] B. Sarasola and K. F. Doerner, "Adaptive large neighborhood search for the vehicle routing problem with synchronization constraints at the delivery location," Networks, vol. 75, 2020.

[17] K. S. J. Glew, S. Wanless, M. P. Harris et al., "Moult location and diet of auks in the North Sea inferred from coupled lightbased and isotope-based geolocation," Marine Ecology Progress Series, vol. 599, 2019.

[18] S. Saeed, M. Asim, T. Baker, and Z. Maamar, "A locationsensitive and network-aware broker for recommending web services," Computing, vol. 101, no. 5, pp. 455-475, 2019.

[19] J.-H. Huh and T.-J. Kim, "A location-based mobile health care facility search system for senior citizens," The Journal of Supercomputing, vol. 75, no. 4, pp. 1831-1848, 2019.

[20] J. D. Oa, "Service quality, satisfaction and behavioral intentions towards public transport from the point of view of private vehicle users," Transportation, vol. 12, no. 2, pp. 134-148, 2021.

[21] B. A. Chang, "Impact of a short-term domestic servicelearning program on medical student education," Annals of Global Health, vol. 85, 2019.

[22] S. Z. Ali and L. Tiziana, "Service utilization patterns for childbirth and neonatal mortality in the Occupied Palestinian Territory during conflict," European Journal of Public Health, vol. 30, no. 5, pp. 856-860, 2020.

[23] N. Wang, "A two-stage charging facilities planning method for electric vehicle sharing systems," IEEE Transactions on Industry Applications, vol. 99, 2020.

[24] B. Luo, X. Li, J. Weng, J. Guo, and J. Ma, "Blockchain enabled trust-based location privacy protection scheme in VANET," IEEE Transactions on Vehicular Technology, vol. 69, no. 2, pp. 2034-2048, 2020.

[25] L. A. Kuehn, S. L. Casperson, J. D. Derner et al., "23 Current progress in the Agricultural Research Service Beef Grand Challenge: a large-scale genetics by environment by management evaluation project," Journal of Animal Science, vol. 98, no. Supplement_4, pp. 13-14, 2020.

[26] C. Zhang, "Geographic image management system based on Silverlight," Haematologica, vol. 99, no. 11, pp. 1057-1062, 2014.

[27] J. Poor, Geographic Image Storage, Transmission and Display System, 2006.

[28] J. A. Santiváñez and E. Melachrinoudis, "Reliable maximinmaxisum locations for maximum service availability on tree networks vulnerable to disruptions," Annals of Operations Research, vol. 286, no. 1-2, pp. 669-701, 2020.

[29] D. Mao, J. Tan, and J. Wang, "Location planning of PEV fast charging station: an integrated approach under traffic and power grid requirements," IEEE Transactions on Intelligent Transportation Systems, vol. 99, pp. 1-10, 2020. 
[30] Y. Wang, "Preserving location privacy in UASN through collaboration and semantic encapsulation," IEEE Network, vol. 99, no. 7, pp. 316-327, 2020.

[31] B. M. Carius, M. D. April, C. S. Pedersen, and S. G. Schauer, "Historical alternans in the emergency department (H.A.t.E.D.) for pain: an analysis of patient pain descriptors to attending and trainee clinicians," Southern Medical Journal, vol. 114, no. 1, pp. 23-27, 2021. 